\title{
D-Space and Deform Closure: A Framework for Holding Deformable Parts
}

\author{
K. "Gopal" Gopalakrishnan and Ken Goldberg \\ IEOR and EECS Departments \\ University of California at Berkeley \\ Berkeley, CA 94720, USA \\ gopal@ieor.berkeley.edu and goldberg@ieor.berkeley.edu
}

\begin{abstract}
We extend the form closure framework for rigid parts to holding a class of deformable parts. In this class, a part is a linearly elastic, frictionless polygon with a finite element mesh and given stiffness matrix. We define the $D$-space (deformation-space) of a part as the C-space of all its mesh nodes. We define the free space as the intersection of the set of topology preserving mesh configurations with the complement of the union of all D-obstacles that represent collisions of the part with finger bodies. Consider a given set of finger bodies in frictionless contact with a part. When positive work is needed to release the part, we say that it is in deform closure. We present a numerical example and prove two results: (1) If contact set holds a rigid part in form closure, it will hold the equivalent deformable part in deform closure and (2) Deform closure is frame invariant.
\end{abstract}

Index Terms - Form Closure, C-Space, Finite Element Methods, Fixturing, Grasping.

\section{INTRODUCTION}

There is a substantial body of research on robotic holding (grasping and fixturing) of rigid objects. Our work is inspired by Rimon and Burdick on rigid body immobility [Rimon96] and by Rimon and Blake [Rimon99] on caging grasps for rigid bodies. We adopt their notation wherever possible.

In C-Space, the part's position and orientation are parameterized in a Euclidian space thus representing the part's configuration in physical space as a point in C-Space (configuration space) [21, 13]. The set of points in C-Space for which the corresponding configuration of the part intersects with a given obstacle is the corresponding Cobstacle. When a part is in contact with an obstacle, its image in C-Space lies on the C-obstacle's surface. The complement of the union of all C-obstacles where the part is allowed to move freely is called the Free Space $\left(C_{\text {free }}\right)$ of the part. Immobility is achieved at a configuration $q_{A}$ if no neighboring configurations in C-Space lie in $C_{\text {free }}$. Unlike rigid parts, deformable parts cannot be fixed in a single configuration. We extend the C-space concept of form closure by defining deform closure.

We model the planar deformable part $E$ by a triangular FEM mesh $M$. In this model, the part perimeter behaves like a planar structure whose perimeter is composed of struts of variable length, with hinges at each vertex. We define the $D$ - space (deformation-space) of $E$ as the $\mathrm{C}$-space of all its mesh nodes.

Given the initial configuration $q_{0}$ of the mesh in Dspace, we define $D_{T}$ as the set of all configurations with the same mesh topology as $q_{0}$. We consider contact with a set of rigid finger bodies $A=\left\{A_{l}, \ldots, A_{k}\right\}$. Each finger defines a D-obstacle $D A_{i}$, the set of mesh configurations that collide with $A_{i}$. The free space $D_{\text {free }}$ is the set of feasible configurations, the intersection of $D_{T}$ with the complement of the union of D-obstacles.

Given part $E$, mesh $M$, FEM stiffness matrix $K$, and contact set $A$, we can determine the potential energy of any point in $D_{\text {free }}$. Given a candidate configuration $q_{A}$, we say that the part can be released from $A$ by a sequence of external wrenches if the part can escape from $q_{A}$. We define $U_{A}$ as the minimum work that needs to be done by these wrenches to release the part from $A$. We say that $A$ holds $E$ in deform closure if $U_{A}>0$.

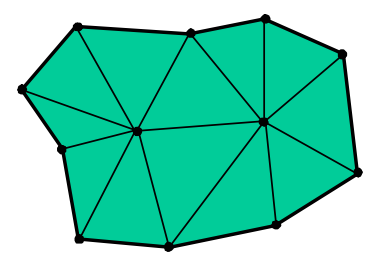

(a)

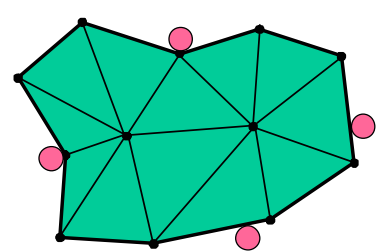

(b)
Fig. 1. (a) Undeformed part. (b) Deformed part held in deform closure by four point contacts.

\section{RELATED WORK}

Bicchi and Kumar provide a concise survey of literature on grasping and fixturing in [1]. Grasps of rigid bodies can be classified as force or form closure. Form closure (immobility) occurs when any neighboring configuration of the part results in collision with an obstacle. Force-closure occurs if any external wrench can be resisted by applying suitable forces at the contacts $[13,19]$.

The mobility of rigid bodies in contact with frictionless finger bodies was initially studied using first order approximations $[17,24,15,12]$. The first order theories are based on approximations of part geometry in infinitesimal neighborhoods and part motion of an infinitesimal length. However, these first order approximations of mobility did

\footnotetext{
* This work is supported in part by the Ford Motor Company URP 2000-403R and NSF Award DMI-0010069.
} 
not always predict immobility correctly.

Rimon and Burdick [18] give rigorous definitions of first and second order immobility. They express paths of the part in free space using functions $q(t)$ based on scalar parameter $t$. They consider the distances $d_{i}$ from the $i^{\text {th }} \mathrm{C}$ obstacle surface to $q(t)$. They then consider the first and second order terms in the algebraic expressions of distances as a function of $t$. The second order terms are needed if first order tests do not show if the distance is increasing or decreasing (the derivative at $t=0$ is 0 ). Geometrically speaking, the first order approximation of immobility is equivalent to approximating the trajectory of the part in Cspace as a straight line, and the surfaces of the C-obstacles as hyperplanes. Second order immobility is equivalent to approximating the trajectory as an elliptical arc and the Cobstacle surfaces as ellipsoids.

Rimon and Blake [20] give a method to find caging grasps, configurations of jaws that constrain parts in a bounded region of $\mathrm{C}$-space such that actuating the gripper results in a unique final configuration. They consider the opening parameter of the jaws as a function of the jaws' positions along the perimeter of the part and use stratified Morse theory to find caging grasps by finding limiting cases that occur when the opening parameter is at a saddle point. [4] also uses the distance function to determine immobile grasps of 2D polygonal parts by a pair of vertical cylindrical jaws engaging the part at its concave vertices.

An efficient geometric algorithm to compute all placements of four frictionless point contacts on a polygonal part that ensure form closure is described by van der Stappen et al [25]. Given a set of four edges, they show how to compute critical contact placements in constant time. The time complexity of their algorithm is bounded by the number of such sets, and runs in an expected time of $O\left(n^{2}\right.$ $\log n$ ) for $n$ vertices. Cheong et al [3] give fast algorithms to find immobilizing grasps of 2D polygonal parts with 2 and 3 contacts. The algorithms find sets of contact wrenches that contain the center of mass. Zhu et al [26] give a grasp metric for 2D and 3D grasps to quantify how firmly the rigid part is held when resisting external forces. Their metric is faster to compute than similar earlier metrics.

Recent work on fixturing deformable and sheet-metal parts is based on the work of Menassa and De Vries [14] where they determine the positions of the primary datum (the datum points needed to locate the part in the correct plane) for 3-2-1 fixturing to minimize deformation. They use a finite element model of the part, and determine fixture locations by optimizing an objective that is a function of the deformations at the nodes. Their work is extended by [16] and [2].

Gopalakrishnan et al [5] propose unilateral fixtures, a new class of fixtures for sheet metal parts with holes, where holding elements lie almost completely on one side of the part, maximizing access for welding, assembly, or inspection. Each primary jaw is cylindrical with a conical groove that provides the equivalent of four point contacts and facilitates part alignment. They present a two-phase algorithm for computing unilateral fixtures where the second phase uses a Finite Element Method (FEM) to compute part deformation and to arrange secondary contacts at part edges and interior surfaces. For a given sheet-metal part, given as a 2D surface embedded in 3D with $e$ edges, $n$ concavities and $m$ mesh nodes, Phase I takes $O\left(e+n^{4 / 3} \log ^{1 / 3} n+g \log g\right)$ time to compute a list of $g$ pairs of primary jaws ranked by quality. Phase II computes the location of $r$ secondary contacts in $O\left(g^{3} r\right)$ time.

$\mathrm{Li}$ et al [10] design fixtures for laser welding using a genetic algorithm within robust design spaces with low to part dimension and jaw location errors. Li et al [11] describe a dexterous part holding mechanism based on vacuum cups and model the elastic deformation of the sheet-metal part using Finite Element Methods and a statistical data model. The results from this model are used to minimize the part's deformation. Shiu et al $[22,23]$ give a heuristic algorithm to analyze the deformation of a sheet metal part by decoupling it into beams based on the part's features, and give an algorithm to allocate tolerances to each feature.

Path planning for elastically deformable parts has been studied using probabilistic roadmaps (PRM). Holleman, Kavraki and Warren [8] give a path planning algorithm for a flexible surface patch. They use a Bezier approximation and an approximate energy function to model deformation of the part. They present experimental results of paths planned for parts generated by a search graph using PRM. Guibas et al [7] improve on the PRM methods for path planning for a surface patch by studying the medial axis of the workspace. Minimum energy configurations of the part are then computed for positions along the axis and connected by quasi-static paths. Lamiraux et al [9] generate a path for a thin rectangular elastic metal plate represented by a Bezier when it is manipulated by constraining the positions and orientations of two opposite edges. Paths are generated using PRM under elastic constraints of the part material.

\section{Deformation-Space}

For this paper, we use a linear FEM model (with linear interpolation, linear elasticity and no mechanical failure). We assume that we are given a polygonal part $E$ with polygonal holes, modeled by a 2D triangular FEM mesh $M$. This FEM model of the part causes the perimeter to behave like a polygon with variable length edges, with hinges at each vertex. An initial undeformed configuration $q_{0}$ of the part is also specified.

Under these assumptions, we construct a Deformation Space (D-Space) $D$ of the part. Depending on the FEM model used, each of the $n$ nodes in $M$ has a predefined number $f$ of degrees of freedom. (These generally consist of translational ( $t$ DOFs) and rotational/torsional ( $r$ DOFs). $)$ Every mesh configuration is specified by specifying each of the $f$ freedoms of each of the $n$ nodes. Given the positions and geometries of all fingers, we represent the mesh in its deformation-space which is the smooth manifold $D=\boldsymbol{R}^{t} \times$ $S O(r) . S O(r)$ is parameterized by $\theta \in \boldsymbol{R}^{r}$ via exponential map parameterization. Thus, for a part with $n$ nodes in $M$, 
each with $f$ degrees of freedom each, the D-space can be parametrized by $\boldsymbol{R}^{n f}$, with each coordinate axis corresponding to one DOF of one FEM node. Any deformed translated or rotated shape of this part can be represented as a unique point in this space. For any point $q$ in $D$, we denote the corresponding shape and configuration of the part by $E(q)$. Note that in our examples with triangular elements with linear elasticity and linear interpolation, $r=0$ and $t=2$.

We define $D_{T}$ as the set of all $q$ that preserve the topology of $M$ identical to the topology at the given undeformed configuration $q_{0}$. Given a finger body $A_{i}$, its corresponding D-space obstacle $D A_{i}$ is the set of all configurations $q$ such that $E(q)$ intersects $A_{i}$.

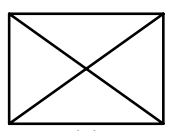

(a)

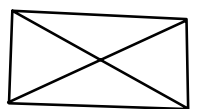

(b)

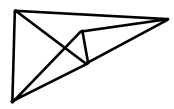

(c)
Fig. 2. For the rectangular part in (a), deformed configuration (b) lies in $D_{T}$, while deformed configuration (c) lies in $D_{T}^{C}$.

$$
\text { We define } D_{\text {free }}=\left(\bigcup_{i=1 . . k} D A_{i}\right)^{C} \cap D_{T} \text { as the set of all }
$$
valid deformable configurations of $M . D_{\text {free }}^{C}$ is the complement of $D_{\text {free }}$.

Examples of configurations that lie in $D_{T}$ and $D_{T}^{C}$ are shown in Figure 2. Figure 3 shows slices of $D_{\text {free }}$ for a rectangular part with 5 nodes and a small circular finger (Figure 3(a)). Each node has 2 translational degrees of freedom about the $\mathrm{x}$ and $\mathrm{y}$ axes. Figure 3(b) assumes nodes 1-4 are fixed, and shows the resulting $D_{\text {free }}$ for node 5 . Figure 3(c) assumes nodes 1, 2, 4 and 5 are fixed, and shows $D_{\text {free }}$ for node 3 .

\section{Potential EnERgy in D-Space}

So far, we have looked at shapes of the part and the mesh that are geometrically possible and may be caused by an arbitrary set of finite wrenches applied on the part. We now include the mechanical properties of the part in our model simply by using the potential energy of any deformed state as predicted by the FEM model.

We assume zero friction between the part and finger contacts, and that the part exhibits perfect linear elasticity with known Young's modulus and Poisson's ratio. Linear FEM analysis with linear interpolation for the given mesh and material properties is assumed to predict the part's deformation accurately.

The nodal displacement vector $X$ is the vector of the displacements of all nodes from their initial positions in configuration $q_{0}$, expressed in the global coordinate frame used to define the FEM model $(M, K, X) . X$ is a distance preserving transformation of $q$ and a special case of expressing $q$ when each node's degrees of freedom are expressed relative to a reference frame whose origin coincides with the mesh node at configuration $q_{0}$, and whose axes parallel to the axes of the global coordinate frame. A deformation potential energy which is a scalar value is given as a function of the deformation of each node. For the linear case with a FEM stiffness matrix of $\mathrm{K}$, the potential energy is represented as $(1 / 2) \mathrm{X}^{\mathrm{T}} \mathrm{K} \mathrm{X}$. The potential energy at a configuration $q$ in $D_{\text {free }}$ is represented as $U(q)$.

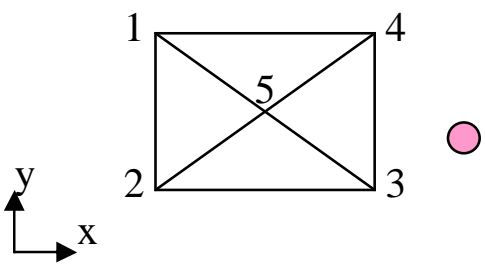

(a)

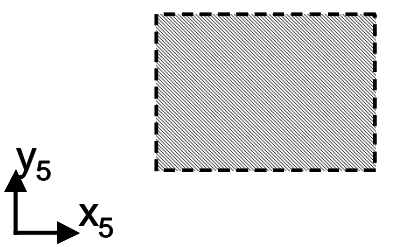

(b)

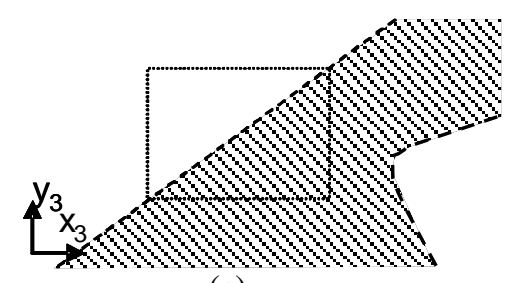

(c)

Fig. 3. For an example part with 5 nodes and one circular obstacle, $D_{\text {free }}$ for nodes 5 and 3 ((b) and (c) respectively) shown when the remaining nodes of the rectangle are held fixed in the positions shown in (a). The shaded region lies inside $D_{\text {free }}$.

\section{Deform Closure}

As input, we consider: 1. the FEM mesh $M$ of the part $E$, 2. stiffness matrix $K$ of the part $E$ corresponding to mesh $M, 3$. the initial configuration $q_{0}$ of the mesh that specifies the initial topology and undeformed mesh shape, 4. set $A$ of frictionless contacts (finger bodies) that represents the candidate fixture, and 5 . the configuration $q_{A}$ in which the part is held by $A$.

We propose to characterize fixtures of $E$ based on the D-Space model with potential energy. We refer to these as deform closure fixtures.

Definition: An equilibrium configuration is any configuration at a local minimum of the potential energy in $D_{\text {free }}$. In the absence of friction and inertial forces, the part comes to rest at an equilibrium configuration.

Definition: We consider a set of wrenches that act on $E$ $\left(q_{A}\right)$ for any equilibrium configuration $q_{A}$. If after the wrenches are applied and then removed, the part may not return to $q_{A}$, the wrenches are said to release $E$.

Definition: If $E\left(q_{A}\right)$ is held by $A$ such that no set of wrenches that increases the deformation potential energy of 
$E$ by at most $U$ can release $E$ from $A$, we say $q_{A}$ satisfies the property stable $(U)$.

In other words, given $U \geq 0$, A holds $E\left(q_{A}\right)$ in stable $(U)$ if and only if $1 . q_{A}$ is at a local minimum of the potential energy in $D_{\text {free }}$, and 2 . We consider all continuous subsets $S$ of $D_{\text {free }}$ such that for all $q_{s} \in S, U\left(q_{s}\right) \leq U\left(q_{f}\right)+U$. For all such subsets $S$, there should not exist $q_{1} \in S$ distinct from $q_{A}$ with $q_{l}$ at a local minimum of the potential energy in $D_{\text {free }}$.

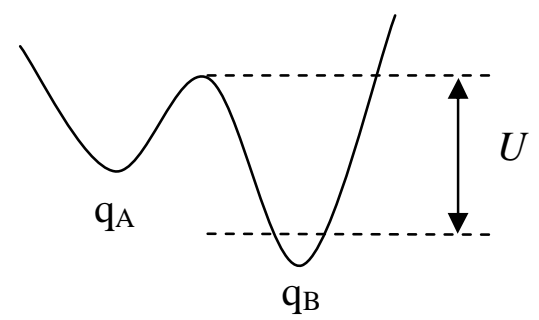

Fig. 4. For the Potential Energy curve shown, $q_{A}$ and $q_{B}$ are stable equilibria. But for the shown value of $U$, only $q_{B}$ satisfies stable (U).

Definition: The threshold potential energy $U_{A}$ for $A$ holding $F$ in configuration $q_{A}$ is defined as $U_{A}\left(q_{A}\right)=\sup \left\{U \mid\right.$ A holding $E\left(q_{f}\right)$ satisfies stable $\left.(U)\right\} \quad$ if $q_{A}$ is an equilibrium configuration, and 0 otherwise. A holds $E\left(q_{A}\right)$ in deform closure if and only if $U_{A}\left(q_{A}\right)>0$.

A configuration with potential energy $U\left(q_{A}\right)+U_{A}\left(q_{A}\right)$ where the part can be released from the deform closure fixture is called its escape configuration. This is inspired by Rimon and Blake's analysis of caging grasps [20]. They define a cage of a part $E$ at configuration $q$ by a k-fingered hand with opening parameter $\sigma$. A configuration $x=(q, \sigma)$ of the part and the hand is a cage if $q$ lies in a connected component of free space $C_{\text {free }}$ which is completely surrounded by the finger $\mathrm{C}$-obstacles $C A_{l}, \ldots C A_{k}$. They define the caging set $C$ as the set of all hand configurations that maintain the part caged between the fingers such that from any initial configuration in $C$, there exists a continuous path in $C$ leading to a desired immobilizing grasp $x_{0}=$ $\left(q_{0}, \sigma_{0}\right)$.

They use stratified Morse theory to analyze cages, and identify maximal caging grasps or puncture grasps as occurring at configurations where the distance between the fingers is at a saddle point.

Similarly in D-space, given a configuration $q_{A}$ that is a strict local minimum of the potential energy, we would like to identify the maximum $U$ for which A holds $E\left(q_{A}\right)$ in deform closure, by identifying an escape configuration near $q_{A}$ that is a saddle point. This value of $U_{A}$ can be used to define a metric for the deform closure fixture. A numerical example appears in the next section.

\section{NUMERICAL EXAMPLE}

We implemented the threshold potential energy used in the definition of deform closure to a simple rubber part modeled by 2 triangular mesh elements and 4 nodes shown in Figure 5(a) and (b). The part is a rhombus and each element an equilateral triangle in the undeformed state. It is held by 4 point contacts, at the midpoint of each edge in Figure 5(b) and near the vertices in 5(a). The FEM model uses plane stress constant strain triangular elements made of an incompressible material (Poisson ratio=0.5). The threshold potential energies for deform closure in 5(a) and 5 (b) are 4 Joules and 547 Joules respectively.

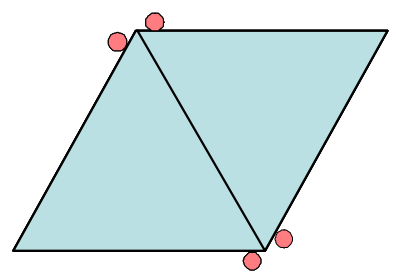

(a)

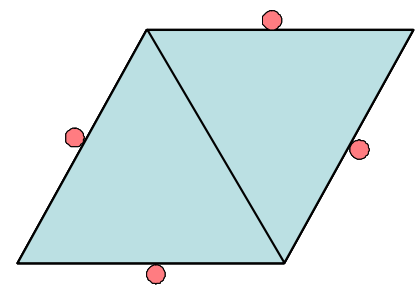

(b)
Fig. 5. Examples of deform closure of a rhombus shaped part with 2 triangular FEM elements. With given mesh and stiffness values, the threshold potential energies needed to release the parts from deform closure grasp (a) is 4 Joules, and from deform closure grasp (b) it is 547 Joules. Thus grasp (b) is preferable under this model.

To determine this threshold configuration, we first list all limiting configurations where a vertex of the part is about to be released from the deform closure fixture. For this example, this happens only when the vertex is in contact with the jaw. Among all such configurations, we considered various cases as to each edge being in contact with or breaking contact with the corresponding jaw. We then determine the configuration with least potential energy among these. Figure 6(d) shows the escape configuration for the deform closure shown in Figure 6(a). This configuration occurs when the deformed part is still a rhombus, but one of the edges lies along 2 jaws (the ones on the left edge and the lower edge), and the opposite edge is in contact with the jaw on the right. One possible path from the configuration in Figure 6(a) to that in 6(d) is through 6(c) and 6(d) with maximum potential energy at $5(\mathrm{~d})$. At no point on this path is the potential energy greater than that at the final configuration.

If the undeformed edges of each triangle were $10 \mathrm{~mm}$ long and the triangle part were $1 \mathrm{~mm}$ thick and made of steel, the limiting threshold potential energy would be 547 Joules.

\section{EQUIVALENCE AND FRAME INVARIANCE THEOREMS}

Given a rigid part, we call an undeformed deformable part with the same shape and configuration of the rigid part its equivalent deformable part.

Theorem 1: If A holds a rigid part in form closure, A will hold the equivalent deformable part in deform closure.

By equivalent, we mean that the undeformed part has the same shape and configuration as the rigid part.

Proof: Since the rigid part in the given configuration is immobile, deformation occurs when the deformable part is perturbed. Since initially the deformation potential energy is at 0 , the potential energy increases to above zero. Thus, $U_{A}>0$. This is true for higher orders of immobility too. 


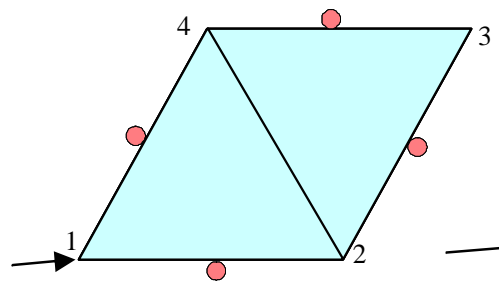

(a)

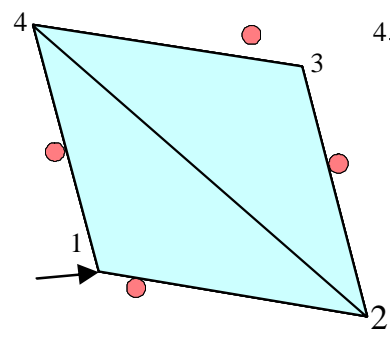

(c)

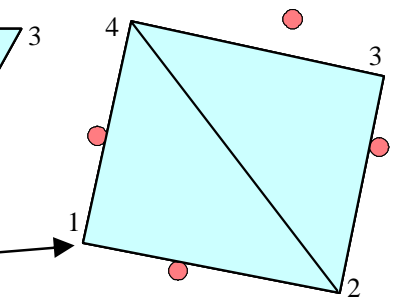

(b)

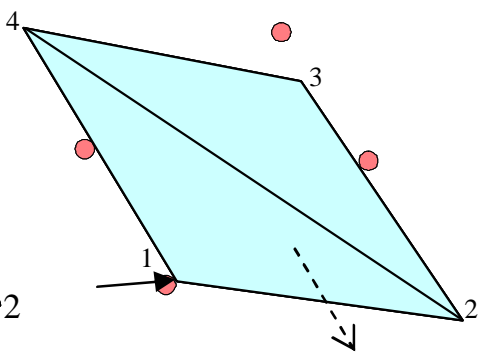

(d)
Fig. 6. An example part in a deform closure fixture (a) and the escape configuration where it is released with minimum increase in potential energy by moving vertex 1 . The escape configuration is shown in (d). One possible path is through configurations (b) and (c). The dashed arrow in (d) shows how the part can be released.

Theorem 2: Frame Invariance.

We now show that the definition of deform closure is frame invariant, i.e. it does not depend on the global reference frame used.

Since the definition of D-space changes with the reference frame used, we prove that the definition of deform closure fixtures does not change with the global reference frame by showing the following:

(a) Displacements caused by a given force are frame invariant

(b) Potential Energy due to a given set of displacements is frame invariant, and

(c) A set of configurations of the part whose image is continuous in $D$-space for one reference frame has an image that is continuous in $D$-space for any other reference frame.

Proof: Let the global stiffness matrix in reference frame $g$ be $K_{g}$. For mesh element $\mathrm{m}_{\mathrm{i}}$, let the component of the stiffness matrix $K$ be $K_{i}$. Let ${ }_{g} T_{i}$ be the transformation matrix between the reference frame of the element and the global reference frame $g$, and $K_{i}$ is the stiffness of element $\mathrm{m}_{\mathrm{i}}$ in the local frame. Thus, the stiffness matrix $K$ is the sum of its components for each mesh element and is given by:

$$
K_{g}=\sum_{i} K_{i}=\sum_{i} T_{i} \cdot K_{i} \cdot{ }_{g} T_{i}^{T} .
$$

Let the reference frames be denoted by subscripts 1 and 2 , and the transformation matrix between the frames be ${ }_{2} T_{1}$. The stiffness matrix changes with the frame used. The two stiffness matrices are related to each other by ${ }_{2} T_{i}={ }_{2} T_{1} \cdot T_{1}$.

Thus:

$$
K_{2}=\sum_{i}{ }_{2} T_{i} \cdot K_{i} \cdot{ }_{2} T_{i}^{T}=\sum_{i}{ }_{2} T_{1} \cdot T_{1} \cdot K_{i} \cdot{ }_{1} T_{i}^{T} \cdot{ }_{2} T_{1}^{T}={ }_{2} T_{1} \cdot K_{1} \cdot{ }_{2} T_{1}^{T}
$$

To prove (a), we consider any given set of forces expressed in reference frame 1 by vector $F_{1}$. Thus, $X_{1}=K_{1}^{-1} \cdot F_{1}$. Expressed in frame 2, the forces are $F_{2}={ }_{2} T_{1} \cdot F_{1}$.

When transformed to reference frame 2, the displacement predicted by frame 1 is:

$$
\begin{aligned}
& X_{2}={ }_{2} T_{1} \cdot X_{1}={ }_{2} T_{1} \cdot K_{1}^{-1} \cdot F_{1}={ }_{2} T_{1} \cdot K_{1}{ }^{-1} \cdot{ }_{2} T_{1}^{-1} \cdot{ }_{2} T_{1} \cdot F_{1} \\
& =\left(\left({ }_{2} T_{1}\right)^{T}\right)^{-1} \cdot K_{1}{ }^{-1} \cdot{ }_{2} T_{1}{ }^{-1} \cdot{ }_{2} T_{1} \cdot F_{1}=\left({ }_{2} T_{1} \cdot K_{1} \cdot{ }_{2} T_{1}^{T}\right)^{-1} \cdot\left({ }_{2} T_{1} \cdot F_{1}\right) \\
& =K_{2}{ }^{-1} \cdot F_{2}
\end{aligned}
$$

This is the displacement predicted by reference frame 2 . Thus, (a) is true.

To prove (b), we consider the potential energy predicted by the reference frame 1 when $E$ is subject to deformations $X_{l}$ is $1 / 2 X_{1}^{T} K_{1} X_{1}$.

The potential energy predicted in frame 2 when $\mathrm{E}$ is subject to the same deformation (but transformed to frame 2) is:

$$
\begin{aligned}
& 1 / 2 X_{2}^{T} \cdot K_{2} \cdot X_{2}=1 / 2\left({ }_{2} T_{1} \cdot X_{1}\right)^{T} \cdot\left({ }_{2} T_{1} \cdot K_{1} \cdot{ }_{2} T_{1}^{T}\right) \cdot\left({ }_{2} T_{1} \cdot X_{1}\right) \\
& =1 / 2 X_{1}^{T} \cdot{ }_{2} T_{1}^{T} \cdot{ }_{2} T_{1} \cdot K_{1} \cdot{ }_{2} T_{1}^{T} \cdot{ }_{2} T_{1} \cdot X_{1}=1 / 2 X_{1}^{T} \cdot K_{1} \cdot X_{1}
\end{aligned}
$$

This is the same as the potential energy in frame 1 . Thus, (b) is proved.

To prove (c), we show that for any 2 configurations of the part, the distance between their images in D-space remains the same in all frames of reference, which implies equivalence in continuity in both frames of reference.

$$
\left|q_{a}-q_{b}\right|=\sum\left(\left(x_{n a}-x_{n b}\right)^{2}+\left(y_{n a}-y_{n b}\right)^{2}\right) \quad \text { summed }
$$
over all nodes $n$, where $x_{n i}$ and $y_{n i}$ are the $\mathrm{x}$ and $\mathrm{y}$ coordinates of node $n$ in configuration $i=a, b$. Since we consider only distance-preserving transformations when changing reference frames, $\left(\left(x_{n a}-x_{n b}\right)^{2}+\left(y_{n a}-y_{n b}\right)^{2}\right)$ is frame invariant for all nodes $n$. Thus, $\left|q_{a}-q_{b}\right|$ is also frame-invariant.

\section{DISCUSSION}

We have presented a new framework for holding deformable parts. We define fingers $A$ to hold part $E$ in deform closure if the increase in potential energy needed to release $E$ from $A$ is non-zero. We propose the minimum amount of work needed to release $E$ as a metric for the quality of a deform closure grasp.

If a model of D-space were constructed by brute force, the amount of computation involved in determining $D_{\text {free }}$ may be extremely high. For example, for the 12 node mesh in Figure 1, the D-Space will be 24-dimensional. However, computing $D_{\text {free }}$ will be simplified to a large extent by the symmetries of D-obstacles and of $D_{T}$. D-obstacles are symmetric, since for any two triangles of the mesh, the collision check for any finger body is identical. Hence, for every triangle in the mesh, the slice of the D-obstacle with fixed positions of other nodes will look identical. Further, the D-obstacle is defined by only the union of these slices, since if any portion of the part collides with the obstacle, at 
least one triangle in the mesh will collide with it. For the part in Figure 1, this causes the D-obstacles to have a 12fold symmetry due to presence of 12 elements in the mesh. Further, for identical obstacles, each D-obstacle is a copy of every other obstacle displaced by a known vector.

Although the set of topology preserving mesh configurations $D_{T}$ may not be symmetric in itself, we can consider a superset $D^{\prime}{ }_{T}$ of $D_{T}$ that consists of all meshes where no triangles intersect and no triangle has zero area. $D^{\prime}{ }_{T}$ contains many configurations that cannot be attained by the physical part (such as a mirror image of the undeformed mesh), but there is never a continuous path in $D^{\prime}{ }_{T}$ connecting the initial mesh to an unattainable mesh. For the part in Figure 1, this results in 23 symmetries because of pairs of distinct triangles, 29 symmetries for pairs of triangles with 2 distinct vertices each, and 13 symmetries for pairs of triangles that have 1 distinct vertex each.

In future work, we will develop algorithms that apply this framework to compute deform closure grasps.

\section{ACKNOWLEDGMENT}

We acknowledge Frank van der Stappen, Rama Koganti, Matt Zaluzec, Ron Alterovitz and Dezhen Song for their invaluable comments and contributions.

\section{REFERENCES}

[1] A. Bicchi and Vijay Kumar, Robotic Grasping and Contact: A Review, Proceedings of IEEE International Conference on Robotics and Automation, pp348-353, 2000

[2] Cai W., Hu S.J., Yuan J.X., Deformable sheet metal fixturing: principles, algorithms, and simulations. Transactions of the ASME. Journal of Manufacturing Science and Engineering, vol.118, (no.3), ASME, Aug. 1996. p.318-24.

[3] Jae-Sook Cheong, Herman J. Haverkort, and A. Frank van der Stappen, On Computing All Immobilizing Grasps of a Simple Polygon with Few Contacts accepted for ISAAC (International Society for Analysis, its Applications and Computation), 2003.

[4] K. "Gopal" Gopalakrishnan, Ken Goldberg, Gripping parts at concave vertices, IEEE International Conference on Robotics and Automation, 2002, Page(s): 1590 -1596, Volume: 2 , 2002.

[5] K. "Gopal" Gopalakrishnan, Matthew Zaluzec, Rama Koganti, Patricia Deneszczuk and Ken Goldberg, "Unilateral" Fixturing of Sheet Metal Parts Using Modular Jaws with Plane-Cone Contacts, Proceedings, IEEE International Conference on Robotics and Automation, pp 39533958, 2003.

[6] K. "Gopal" Gopalakrishnan and Ken Goldberg, D-Space and Deform Closure: A Framework for Holding Deformable Parts, IEEE International Conference on Robotics and Automation (ICRA), May 2004. This paper is available at http://ieor.berkeley.edu/ goldberg/pubs

[7] Guibas, L.J.; Holleman, C.; Kavraki, L.E.; A probabilistic roadmap planner for flexible objects with a workspace medial-axis-based sampling approach, Intelligent Robots and Systems, 1999. IROS '99. Proceedings. 1999 IEEE/RSJ International Conference on, Volume: 1 , 17-21 Oct. 1999, Page(s): 254 -259 vol.1.

[8] Holleman, C.; Kavraki, L.E.; Warren, J., Planning paths for a flexible surface patch, Robotics and Automation, 1998. Proceedings. 1998 IEEE International Conference on , Volume: 1 , 16-20 May 1998, Page(s): 21 -26 vol.1.

[9] Lamiraux, F.; Kavraki, L.E.; Path planning for elastic plates under manipulation constraints, Robotics and Automation, 1999. Proceedings. 1999 IEEE International Conference on , Volume: 1, 1015 May 1999, Page(s): $151-156$ vol.1.

[10]Li B., Shiu B.W., Lau K.J., Fixture configuration design for sheet metal assembly with laser welding: a case study. International Journal of Advanced Manufacturing Technology, vol.19, (no.7), SpringerVerlag, 2002. p.501-9.

[11]Li H. F., Ceglarek D., Shi J., A Dexterous Part-Holding Model for Handling Compliant Sheet Metal Parts, ASME Transactions, Journal of Manufacturing Science and Engineering. Vol. 124, No. 1, pp. 109$118,2002$.

[12]X. Markenscoff, L. Ni and C. H. Papadimitriou, The Geometry of Grasping, International Journal of Robotics Research, Vol. 9, No. 1, pp 61-74, 1990.

[13]Mason M.T., Mechanics of Robotic Manipulation, The MIT Press, 2001.

[14]Menassa R., De Vries W., Optimization Methods Applied to Selecting Support Positions in Fixture Design, ASME Journal of Engineering for Industry, vol 113, pp. 412-418, 1991.

[15]B. Mishra, J. Schwarz, and M. Sharir, On the existence and Synthesis of Multifinger Positive Grips, Algorithmica 2, 1987.

[16]Rearick M.R., Hu S.J., Wu S.M., Optimal Fixture Design for Deformable Sheet Metal Workpieces, Transactions of NAMRI/SME, vol. XXI, pp. 407-412.

[17]F. Reuleaux, The Kinematics of Machinery. New York: Macmillan 1876, republished by New York: Dover, 1963.

[18]Rimon E. and Burdick J., On force and form closure for multiple finger grasps, Proceedings of IEEE International Conference on Robotics and Automation, 1996, pp. 1795 -1800 vol.2.

[19]Elon Rimon and Joel Burdick, Mobility of bodies in contact - I, IEEE transactions on Robotics and Automation, 14(5): 696-708, 1998.

[20]Rimon, E. and Blake, A., Caging planar bodies by one-parameter two fingered gripping systems, International Journal of Robotics Research, v18, n3, March 1999, pp. 299-318.

[21] J. M. Selig. Geometrical Foundations of Robotics. World Scientific, 2000.

[22]Shiu, B.W., Ceglarek, D., Shi, J., 1997 "Flexible Beam-Based Modeling of Sheet Metal Assembly for Dimensional Control," Trans. of NAMRI, Vol. XXV, pp. 49-54.

[23]Shiu, B.W., Apley, D., Ceglarek, D., Shi, J., 2003 "Tolerance Allocation for Sheet Metal Assembly using Beam-Based Model", Trans. of IIE, Design and Manufacturing,, Vol. 35, No. 4, pp. 329-342. (April 2003). The paper was also summarized and featured in the March 2003 edition of Industrial Engineer (formerly IIE Solutions).

[24]P. Somoff, Uber gebiete von schraubengeschwindigkeiten eines starren korpers bieverschiedener zahl von stuz achen, Zeitschrift fur Mathematic and Physik, vol. 45, pp. 245-306, 1900.

[25]Van der Stappen A.F., Wentink C., Overmars M.H., Computing formclosure configurations, Proceedings of IEEE International Conference on Robotics and Automation, vol.3, pp. $1837-1842,1999$.

[26]Zhu X., Ding H., Wang J., Grasp analysis and synthesis based on a new quantitative measure, IEEE Transactions on Robotics and Automation, vol. 19, no. 6, pp. 942-953, Dec. 2003. 